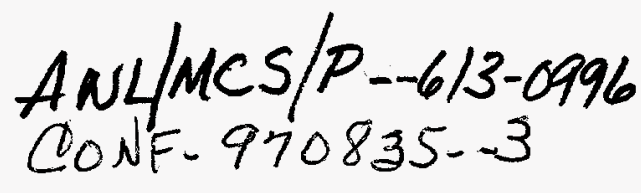

\section{A Secure Communications Infrastructure for High-Performance Distributed Computing}

The submitted manuscript has been created by the University of Chicago as Operator of Argonne National Laboratory ("Argonne") under Contract No. W-31-109-ENG-38 with the U.S. Department of Energy. The U.S. Government retains for itself, and others acting on its behalf, a paid-up, nonexclusive, irrevocable worldwide license in said articie to reproduce, prepare derivative works, distribute copies to the public, and perform publicly and display publicly, by or on behalf of the Government.

\author{
Ian Foster, ${ }^{1}$ Nicholas T. Karonis, ${ }^{3}$ Carl Kesselman, ${ }^{2}$ Greg Koenig, ${ }^{1}$ Steven Tuecke ${ }^{1}$ \\ ${ }^{1}$ Mathematics and Computer Science \\ Argonne National Laboratory \\ Argonne, IL 60439 \\ ${ }^{2}$ Information Sciences Institute \\ University of Southern California \\ Marina del Rey, CA 90292
}

${ }^{3}$ High-Performance Computing Laboratory

Department of Computer Science

Northern Illinois University

DeKalb, IL 60115

http://www.globus.org/

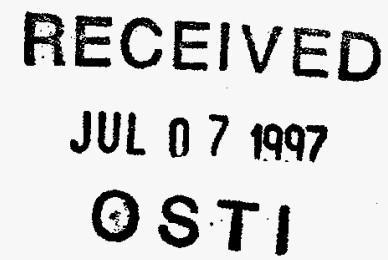

\begin{abstract}
Applications that use high-speed networks to connect geographically distributed supercomputers, databases, and scientific instruments may operate over open networks and access valuable resources. Hence, they can require mechanisms for ensuring integrity and confidentiality of communications and for authenticating both users and resources. Security solutions developed for traditional client-server applications do not provide direct support for the program structures, programming tools, and performance requirements encountered in these applications. We address these requirements via a security-enhanced version of the Nexus communication librar; which we use to provide secure versions of parallel libraries and languages, including the Message Passing Interface. These tools permit a fine degree of control over what, where, and when security mechanisms are applied. In particular, a single application can mix secure and nonsecure communication, allowing the programmer to make fine-grained security/performance tradeoffs. We present performance results that quantify the performance of our infrastructure.
\end{abstract}

\section{Introduction}

Recent developments in networking are enabling the construction of high-performance distributed computing applications that span supercomputers, large-scale database sys- tems, specialized scientific instruments, and other resources located at many sites [2]. These resources may be connected by dedicated or shared high-speed networks, and programs often must achieve a substantial fraction of peak computer and network performance. The more than 60 groups participating in the I-WAY wide-area computing experiment [3] demonstrated the wide range of applications that can fit this general framework, including scientific simulation, collaborative engineering, and computer-enhanced instrumentation.

Inspired by the promising results obtained in early experiments, various groups are developing more substantial high-performance distributed computing testbeds and applications. However, their ability to use these systems for production purposes depends crucially on the availability of appropriate security mechanisms. Owners of resources require authentication mechanisms to protect themselves against malicious users. Users of resources may also demand authentication of resources, in order to protect themselves against spoofing by malicious resource providers. Users will often need to ensure that the integrity and confidentiality of data communicated between resources are not compromised, particularly when communication occurs over public networks. Other forms of attack can also be of concern, such as denial of service attacks against applications that use supercomputers to control remote devices.

The task of meeting these security requirements is complicated by the distinctive program structures, computing environments, and performance requirements encountered in high-performance systems. Traditional distributed systems 


\section{DISCLAIMER}

This report was prepared as an account of work sponsored by an agency of the United States Government. Neither the United States Government nor any agency thereof, nor any of their employees, make any warranty, express or implied, or assumes any legal liability or responsibility for the accuracy, completeness, or usefulness of any information, apparatus, product, or process disclosed, or represents that its use would not infringe privately owned rights. Reference herein to any specific commercial product, process, or service by trade name, trademark, manufacturer, or otherwise does not necessarily constitute or imply its endorsement, recommendation, or favoring by the United States Government or any agency thereof. The views and opinions of authors expressed herein do not necessarily state or reflect those of the United States Government or any agency thereof. 


\section{DISCLAMMIER}

Portions of this document may be illegible in electronic image products. Images are produced from the best available original document. 
often have a client-server structure, with limited mutual trust between client and server. In contrast, parallel programs may comprise hundreds or thousands of tightly coupled, fully trusting processes. Distributed systems employ remote procedure call (RPC) or TCP/P as their primary communication mechanism. In contrast, the applications that we consider here may communicate by using two-sided message passing, streaming protocols, multicast, and/or single-sided get/put operations, as well as RPC; furthermore, they are typically programmed by using message-passing libraries such as the standard Message Passing Interface (MPI [11]) or with specialized parallel languages (e.g., HPF [15] or $\mathrm{HPC}++$ ). Programs must run on parallel computers, which typically provide specialized mechanisms for process creation, communication, and so forth, and which may even run specialized operating systems.

Historically, we find that security technologies are used only if they are incorporated into common tools in a seamless and painless fashion. In the case of high-performance computing, this suggests a need for secure versions of parallel programming tools such as MPI. These security enhanced tools must support the diverse process creation and authentication mechanisms encountered in high-performance systems, and must address scalability issues that arise when dealing with hundreds or thousands of processes. In addition, the demanding performance requirements of highperformance applications introduces a need for mechanisms that provide programmers with fine-grain control over what, when, and where security mechanisms are used in programs.

We are developing a secure communications infrastructure that addresses these various concerns. This infrastructure builds on existing components and standards whenever possible (e.g., SSL [12], Kerberos [25], GSS-API [17]), while also extending the state of the art to provide four new capabilities:

- A secure process creation interface that supports the wide range of process creation mechanisms encountered in high-performance computing systems, and that addresses scalability issues that arise in programs that may need to create hundreds or thousands of processes.

- Techniques for managing the use of multiple security mechanisms within a single application, in a way that provides a uniform high-level programming model while allowing the choice of low-level security mechanism to vary according to what is communicated, where it is communicated, and when it is communicated.

- Techniques for managing the transfer of secure logical communication links among processes in large-scale distributed computations.

- Security-enhanced implementations of multiple parallel libraries (MPI, CAVEcomm, etc.) and languages
(HPF, HPC ++ , etc.), that enable programmers to use our secure process creation and communication mechanisms while using familiar tools.

These new capabilities have been implemented and evaluated in the context of Nexus [10], a low-level multithreaded communication library designed to support highperformance communication in heterogeneous environments. (The security-enhanced libraries and languages referred to above are all layered on top of Nexus.) While these capabilities are not in themselves a complete solution to the problem of providing security in high-performance distributed applications, we do believe that they represent useful steps towards that goal.

The rest of this paper is as follows. In Section 2, we introduce the problems that we seek to address in our work. In Section 3, we provide an overview of our approach and review the Nexus communication infrastructure. In Sections 5 and 6 , we describe our secure communications infrastructure, and in Section 7, we present some experiments that allow us to evaluate its effectiveness. Finally, in Sections 8 and 9 we discuss related work and present our conclusions, respectively.

\section{Requirements}

We are interested in applications that integrate geographically distributed computing, network, information, and other systems to form "virtual" networked computational resources. For example, global climate scientists often employ large coupled simulation models, constructed by linking models of atmospheric and ocean behaviors. Such coupled models may use multiple supercomputers to exploit large aggregate memory or to run different components more quickly on different architectures [19, 23]. High-end collaborative engineering environments connect supercomputers, databases, and advanced display devices to provide remote access to shared state, which may include simulated entities as well as people $[4,5]$. "Smart instruments" connect scientific instruments or other data sources to remote computing capabilities [16]. In each case, computations span heterogeneous collections of resources, often located in multiple administrative domains. They may involve hundreds or even thousands of processes. Communication costs are frequently critical to achieved performance, and programs often use complex computation/communication structures to reduce these costs.

The development of a comprehensive solution to the problem of ensuring "security" in such applications is clearly a complex and multi-faceted problem. In this article, we focus our attention on two significant subproblems, namely the authentication of users and resources when creating computational entities ("processes") on local and/or remote com- 
puter systems (the process creation problem), and the assurance of integrity and confidentiality when exchanging data between these processes (the communication problem).

\subsection{Process Creation}

We use the term process creation to refer to the mechanism by which computational resources are integrated into computations. These resources may all be acquired before the computation starts (i.e., static allocation) or may be acquired and released during the course of the computation (dynamic allocation). Computational resources of interest include both single-processor and multiprocessor systems, and the low-level mechanisms used to initiate computation may be quite different in each case. For example, on a workstation we might use secure or unsecure "remote shell" (rsh) mechanisms or hand-crafted process creation servers; in contrast, parallel computers typically provide specialized mechanisms that start a user-supplied executable on multiple processors and may require interfacing with local resource management systems such as a partition manager or scheduler [13].

A secure process creation facility for high-performance programs must support a heterogeneous mix of process creation mechanisms. It should support authentication of the user of remote resources and/or of the resources themselves. It also needs to provide for the establishment of the security contexts required for subsequent secure communication within the program. Because a computation may comprise hundreds or thousands of processes, which typically are mutually trusting once created, it is both impractical and unnecessary to perform a formal authentication process between every pair of processes. Instead, we need scalable mechanisms for process creation that allow a process to transfer to its offspring the right to communicate with other processes in a computation.

\subsection{Communication}

Once processes have been created, they need to be able to exchange data and synchronize their execution. As noted above, the applications in which we are interested communicate by using a variety of interaction mechanisms. Communication performance is often critical, but as messages are often small, latency can be as important as bandwidth. Collective communication operations across multiple processes can exacerbate the impact of latency on performance. Furthermore, performance and functionality requirements frequently motivate the use of multiple low-level communication methods within a single application. For example, coupled models often need to use machine-specific communication methods within computers and optimized wide area protocols between computers $[19,23]$. Collaborative environments require a mixture of protocols providing different combinations of high throughput, multicast, and high reliability $[4,5]$. Smart instrument applications may need to be able to switch among alternative communication substrates in the event of error or high load [16]. In general, the method used for a communication can vary according to where communication is being performed, what is being communicated, or when communication is performed [8].

These considerations place demanding requirements on a secure communications infrastructure. It is clearly critical to be able to specify the security mechanism used for a particular communication independently of the low-level method used to achieve that communication. More challenging perhaps is that programmers must be able to write programs that mix secure and unsecure communication. For example, let us consider a coupled climate model as a prototypical scientific simulation for which security mechanisms may be required. (While this example may appear contrived, the controversy that surrounds global change studies suggests that security could well be a concern, if computing in an open environment.) Assume that the model runs the ocean and atmosphere model components on two separate IBM SP2 parallel computers, connected by an open highspeed network. The programmer writes the coupled model so that all communication is expressed using MPI; the MPI implementation selects communication methods for each message, according to message destination [9]. Communication between two nodes in the same SP2 takes place over a dedicated, high-speed switch using IBM-specific protocols, and as this environment is tightly controlled, we might reasonably decide that security measures such as encryption are not required. In contrast, communication between two nodes in different SP2s occurs over a general purpose computer network using TCP/IP, and may well require security measures. In Section 7, we present performance results that demonstrate the advantages of applying security mechanisms only between models.

In this example, it is sufficient to select security mechanisms according to where communication is directed: that is, according to the underlying physical communication structure. In other situations, we believe that it is important that programmers be able to vary security mechanisms according to the logical communication structure of a program. For example, we may want to use different security mechanisms for communications representing "control" and "data."

\section{Our Approach}

We seek to address the requirements outlined in the preceding section by constructing a secure communications infrastructure based on a portable communications library called Nexus [10]. We chose to work with Nexus for two reasons. First, it supports many of the tools that are commonly 
used for application development in parallel and distributed systems, such as the Message Passing Interface (MPI) [11], High Performance Fortran (HPF) [15], and CAVEcomm [5] (a specialized library for collaborative environment applications). Second, its architecture has been designed to support the coexistence and concurrent use of different process creation and communication methods [8]. The latter feature simplifies the integration and management of different security methods.

Figure 1 shows some of the parallel tools that have been constructed with Nexus mechanisms. Each of these libraries or languages use Nexus facilities to create processes and to exchange data between processes; Nexus handles automatically the various low-level issues relating to the process creation and communication methods to be used in different situations.

\subsection{Nexus Structure}

The Nexus communication library is structured in terms of five basic abstractions: nodes, contexts, threads, communication links, and remote service requests. A computation executes on a set of nodes and consists of a set of threads, each executing in an address space called a context. (For the purposes of this paper, it suffices to assume that a context is equivalent to a process.) An individual thread executes a sequential program, which may read and write data shared with other threads executing in the same context. Inter-context references called communication links provide a global name space for objects, while the remote service request ( $\mathrm{RSR}$ ) is used to initiate communication and invoke remote computation. Nexus support for threads is relevant to this paper to the extent that threads can be an important latency hiding device, and multithreading can have implications for how we maintain and use security information.

In the following, we expand upon two aspects of the Nexus system: communication links and management of multiple communication methods.

\subsection{Communication Links}

As illustrated in Figure 2, communication links connect data structures called startpoints and endpoints. (Prior papers on Nexus [10] referred to communication links as global pointers; we adopt the alternative terminology to emphasize that we are not assuming a global address space.) A communication link is formed by binding a startpoint to an endpoint. Many startpoints can be bound to a single endpoint and there can be many startpoints and endpoints within a process.

Nexus supports a single communication operation: the remote service request, or RSR. An RSR is directed from a startpoint to an endpoint, causing the transfer of data from the startpoint process to the endpoint process and the remote

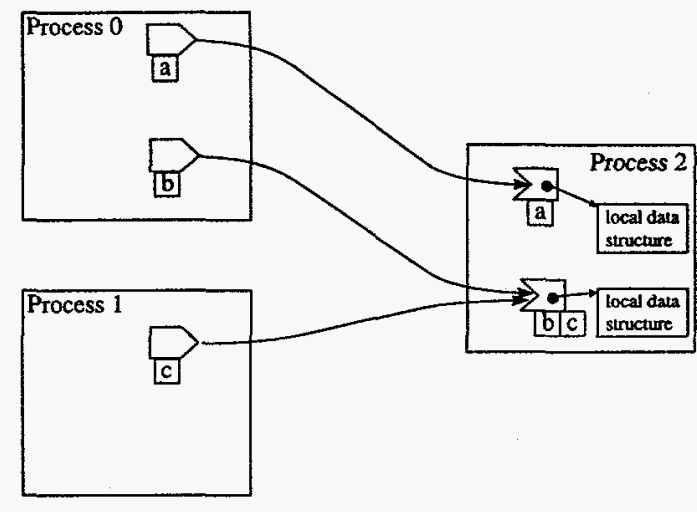

Figure 2. The Nexus secure communications infrastructure. The figure shows three startpoints (in the two processes on the left) referencing two endpoints (on the right). The boxes labeled " $a$," " $b$," and "c" are security contexts; these are discussed below.

execution of a function specified to be an endpoint handler. An advantage of the startpoint construct in a distributed computing environment is that the startpoint can be used to encapsulate not only information about where a remote object is located, but also how to communicate with that remote object. This feature has been exploited to manage the use of multiple communication methods [8].

The endpoint construct allows us to associate local state with the remote location referenced by a startpoint. This state can be used to maintain security information, and hence is valuable when implementing stream-oriented communication routines, such as encryption based on stream ciphers. As illustrated in Figure 2, multiple versions of this local state can be maintained, one for each startpoint in the case where multiple startpoints are associated with a single endpoint.

A startpoint/endpoint pair represents a simplex communication channel: that is, it specifies a remote destination to which a communication operation can be directed by an RSR. These channels can be created dynamically; once created, a startpoint (but not an endpoint) can be communicated between nodes by including it in an RSR message buffer. Hence, a startpoint can be thought of as a capability granting rights to operate on the associated endpoint. The RSR mechanism allows point-to-point communication, remote memory access, streaming protocols, and multicast to be supported within a single framework. 


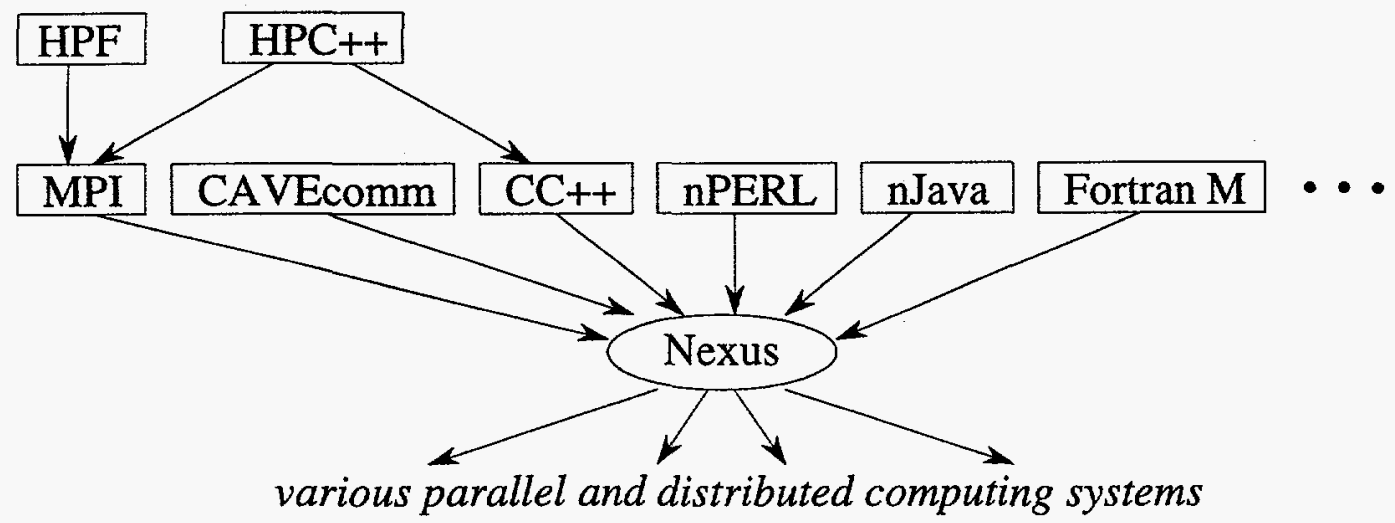

Figure 1. The Nexus communication infrastructure

\subsection{Communication Method Selection}

As noted earlier, high-performance applications can require the use of different communication mechanisms in different situations. Nexus incorporates automatic configuration mechanisms that allow it to use configuration information provided by a Metacomputing Directory Service (MDS) [7] to determine which startup mechanisms, network interfaces, and communication methods to use in different situations [8]. These mechanisms allow Nexus programs to execute unchanged in different environments, with communication methods selected according to default rules, depending on the source and destination of the message being sent. For example, automatic selection allows Nexus RSRs to use IBM's Message Passing Library (MPL) within an IBM SP2 and TCP/IP between computers. Manual selection is also supported. for example allowing selection of specialized ATM protocols when appropriate. In each case, selection mechanisms are employed whenever a startpoint is received from another process, and hence apply both during initial process creation and subsequently as additional communication links are established.

\section{Security Contexts}

As we describe in the next two sections, we extend Nexus in two main ways to develop our secure communications infrastructure. First, we define a secure process creation interface, and integrate this with Nexus process creation mechanisms. Second. we extend Nexus communication mechanisms to use and manage security information. Both extensions make extensive use of a security context similar to that used in GSS-API [17].

The Nexus security context is a data structure used to encapsulate security information. Security contexts are as- sociated with communication links and al ways exist in pairs: one context is stored in a startpoint and the other in the associated endpoint. Each security context is composed of two parts: the security configuration and the security state. The security configuration describes what type of security should be used for the communication link as well as the manner in which those security measures should be applied. For example, we might configure one security context so that each message is encrypted using DES/ECB, while another security context is configured to encrypt with $\mathrm{RC} 4$ and also to perform authentication. This design not only allows us to configure security characteristics on a per-link basis, but also provides a framework in which we may exploit different implementations of security algorithms within a single application, e.g., exploiting high-performance encryption hardware [26] that only exists on some machines.

The security state houses the values needed to enforce the security specified by the configuration, such as keys and initialization vectors. Some encryption algorithms change the values of these keys and/or initialize vectors as a function of the plaintext they encrypt and ciphertext they decrypt. For those security states that change over time, endpoints are required to maintain a distinct security state for each startpoint bound to them. This necessitates additional communication when startpoints are copied (a round trip message) or destroyed remotely.

\section{Process Creation}

Parallel programs use process creation mechanisms to initiate computation on other computers. In Nexus, process creation involves a call to a "create process" function, which invokes machine-specific mechanism to create the new process and instantiates a startpoint referencing an endpoint in the newly created context. Subsequent communication with 


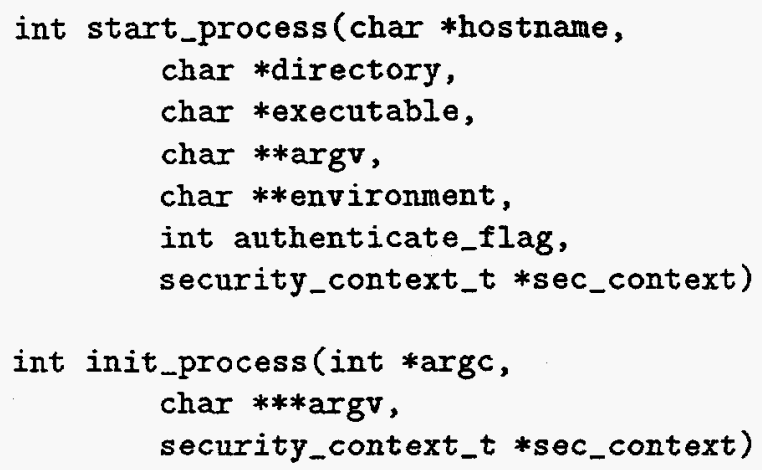

\section{Figure 3. Functions used to add a new pro- cess to a Nexus computation.}

that context occurs over the new communication link. The same interface is used to create multiple contexts (for example, when initiating computation on a parallel computer), except that the call returns a vector of startpoints, one per new process.

Typically, process creation involves interaction with some remote service, whether this be an rsh daemon, a scheduler on a supercomputer, or some other specialized server. Authentication of the requester and/or the remote server may be required, and an initial security context must be established for subsequent communication between requester and newly created process. As noted previously, we need to deal with a wide variety of process creation and authentication mechanisms, and must address scalability issues that arise when creating large number of processes.

\subsection{Interface}

We address the need to deal with a wide variety of process creation and authentication mechanisms by defining a standard interface. Two of the functions in this interface are start_process and init process (Figure 3). Process creation is initiated by a call to start_process. Using the Metacomputing Directory Service, start_process can determine the authentication protocols that are acceptable to the specified host. Based on this information, the initiating process selects the appropriate authentication service and contacts this service to initiate process creation. Depending on the value of the supplied authentication flag and the requirements of the host being contacted, authentication may be required just for the client, or for both the client and the server. An initial security context can be provided to the start_process call; this is encoded as a byte array, passed over a secure channel, and made available to the newly created process by placing it in an environment variable.
Successful authentication results in the creation of a new process on the specified host, with directory, executable, arguments, and environment as specified in the start_process call. The newly created process must call the init process function before performing other computation. The call returns the process arguments (argc, argv) and populates a user-supplied security context with the one provided by the process that called start process.

The two functions just described allow us to create a set of processes and an initial set of shared security contexts. The Nexus implementation then completes the negotiation process by using these shared security contexts to establish an initial communication link (and associated security context) from the requesting process to the created process. Note that subsequent communication with the newly created process can occur with any communication mechanism supported by Nexus (TCP, vendor-specific libraries, etc.). The interface also includes a split-phase version of the start_process function, so that multiple process creation requests can proceed concurrently.

\subsection{Implementation Examples}

Implementations of the process creation interface require mechanisms for authenticating the user and/or the process creation servers, and for establishing a secure channel for the exchange of the initial security context. We have developed a variety of such implementations. As an example, we consider a Secure Socket Library (SSL)-based process creation server. This acts as an SSL server, while the process calling start process (the creating process) acts as an SSL client. The client connects to the server using normal SSL mechanisms, thus performing authentication and establishing a secure channel between the client and server. The client then uses this channel to pass the various process creation arguments to the server, which creates the new process. When the new process calls init_process, it configures itself using the passed command line arguments, and initializes its security context argument using the information passed to it by the server in environment variables. This negotiation process completes with a communication link (and associated security context) being created from the requesting process to the created process.

As a second example, we consider what happens when we need to create many processes at a remote location. One approach would be to make multiple start_process requests to the appropriate remote server. However, this approach has significant scalability problems. Hence, we instead use a single request to ask that multiple processes be created. The process creation server then creates the processes independently, accumulating the startpoints as they become available; when it is done, it returns the vector of startpoints to the requesting process. Note that no additional 
authentication is required when transferring the startpoints (and associated security contexts) from the "proxy" node to the requesting process, because we assume that processes in a parallel program are mutually trusting. These mechanisms allow a program to create large numbers of processes quickly, by using a hierarchical process structure.

We are currently engaged in recasting these and other implementations in terms of the functions provided by GSSAPI [17], with the goal of simplifying code and promoting reuse.

\section{Communication}

As described above, Nexus allows security contexts to be associated with communication links. This structure gives the tool developer (or application programmer) a fine degree of control over how security mechanisms are applied during communication. Different contexts can be associated with different links; in particular, some links may not have any security context at all. Critical to the success of this strategy is that links that do not require security do not have to pay a performance penalty.

Figure 2 shows how startpoints and endpoints are extended with security contexts. In this figure, the boxes labeled " $a$," "b," and "c" represent security contexts. Notice that the lower endpoint (on the right) has two security contexts associated with it, one for each associated startpoint. This ability to associate multiple security contexts with an endpoint is important for several reasons. First, different startpoints might communicate by using different security mechanisms; second, even if they use the same security mechanism, multiple security contexts are required when using encryption mechanisms (e.g., DES stream ciphers) that update the security state as a function of the previously encrypted plaintext.

Nexus mechanisms that manipulate startpoints and endpoints are extended to deal with security contexts. Whenever a startpoint is copied or sent to another process as part of an RSR (hence establishing a new communication link), a new pair of security contexts is created. Depending on the type of security context being created, the copy operation may require communication with the endpoint, requiring a round trip communication.

The application of security mechanisms when initiating or receiving an RSR is triggered by an "escape" tag associated with a Nexus startpoint and endpoint. If this escape tag is set, a specified security transformation is applied to communicated data. At the endpoint, we must identify the correct security context for the incoming communication. To facilitate this, we must place a context identifier in the message header. Exchanging the context identifier is one reason why copying a security context may require communication with the endpoint.
The mechanisms just described have the desirable property of introducing little unnecessary overhead, particularly in the case when they are not used. When they are used, costs associated with this mechanism (relative to a communication method that always performs encryption, for example) are a test on the "escape" flag followed by a lookup of a small table to see what transformation should be applied. If a startpoint is replicated, a small security context index must be included in each RSR. Space overhead comprises the encoding of the security context. When not in use, the only time overhead is the test on the escape flag; there is no space overhead. See Section 7 for additional discussion of performance.

Nexus constructs a remote service request by a series of "put" calls (used to designate the data to be transferred) followed by a "send" (which completes the transfer). Our current security-enhanced Nexus copies data into a contiguous buffer, to which a single encryption call is applied. An alternative approach is to incorporate encryption operations in the "put" calls, hence reducing the number of times that data is copied. We have experimented with both approaches, and find that for DES/ECB the latter approach is typically 5-7 percent faster. The difference would be larger for lowercost encryption techniques.

\subsection{Logical Connections}

Because security mechanisms are integrated into Nexus at a low level, they need not be visible to the programmer. That is, it is straightforward to configure a Nexus application (and hence an application code using any of the various libraries or languages layered on Nexus) so that all communications are secured using the same standard mechanism. Furthermore, this security need not interfere with the various communication optimizations incorporated in Nexus. For example, in a heterogeneous environment, Nexus can, as usual, use TCP/IP between parallel computers and vendorsupplied communication libraries or shared memory within parallel computers.

Nevertheless, the full power of our architecture becomes apparent when the programmer (or tool developer) wants to implement more sophisticated communication structures. Because security contexts are associated with startpoints and endpoints, rather than processes, we can maintain multiple logical connections between a pair of processes, and associate different security mechanisms with different connections. This capability allows the programmer to apply security mechanisms selectively, depending on what is being communicated, where it is being communicated, and even on when communication is performed. For example, we may protect the integrity of control messages at all times, but encrypt data messages only when these are passed over open networks; or we can use specialized encryption techniques 
for particular types of data $[18,1]$. Note that because security context information is associated with communication links, not communication calls, the code that actually performs communication does not need to be aware of whether security mechanisms are being applied.

The ability to associate security contexts with logical connections is particularly useful in multithreaded environments, where communications over different logical connections can be interleaved at the physical level. The Nexus architecture avoids the need for an additional layer of multiplexing/demultiplexing, as would be required, for example, if all communications between two processes had to occur within a single stream cipher-based security context.

A number of approaches can be taken to specifying the security contexts that are to be used for specific communications. As noted above, Nexus mechanisms provide a degree of automatic management. Once a startpoint/endpoint pair has been created, the startpoint can be communicated to other processes, and any process receiving the startpoint can then communicate securely with the original process, by using the startpoint and its associated security mechanism. For more fine-grain control, Nexus provides functions for setting the security attributes of a startpoint and endpoint. Libraries layered on top of Nexus can use other, higher-level mechanisms. For example, an MPI implementation can associate security attributes with a communication structuring mechanism called communicators [11].

\section{Experimental Results}

We report on a number of experiments that we have conducted to study the performance of our techniques. These comprise a simple microbenchmark, designed to yield insights into the costs associated with basic communication operations, and a large-scale application study. We emphasize that these experiments have all been performed in the context of a large-scale working system.

All experiments are performed on the Argonne IBM SP2, which connects 64 Power 1 processors with an SP2 high-speed switch. The SP2 supports both a fast, machinespecific communication library (MPL) and TCP/TP. MPL has performance characteristics typical of high-speed parallel computer communication libraries ( $35 \mathrm{MB} / \mathrm{sec}$ bandwidth, small-message latencies of around $100 \mu \mathrm{sec}$ ). TCP over the $\mathrm{SP} 2$ switch runs at about $8 \mathrm{MB} / \mathrm{sec}$ and incurs small-message latencies of around $2 \mathrm{msec}$; hence, it has performance characteristics similar to a tuned OC3 or faster ATM network in a metropolitan area network. TCP communication on the SP2 that does not used the high-speed switch uses Ethernet.

\subsection{Microbenchmark Results}

We use a microbenchmark to compare the performance of secure and unsecure versions of our basic communication mechanisms. This Nexus program bounces a vector of fixed size back and forth between two processors a large number of times. Each communication is achieved by an RSR to the remote node, with the RSR handler that executes on the remote node invoking an RSR back on the originating node. The experiment is repeated for different vector sizes. Figures 4 and 5 show results obtained in five different configurations: Nexus when using IBM's low-level MPL communication library, with and without DES encryption and MD5 authentication (MPL Secure, MPL Unsecure); Nexus when using TCP/IP communication, with and without DES encryption and MD5 authentication (TCP Secure, TCP Unsecure); and Nexus when using SSL over TCP, with DES encryption and MD5 authentication (TCP/SSL Secure). In those experiments that used TCP/IP communication (TCP Secure, TCP Unsecure, and TCP/SSL Secure) we did not utilize the SP2 high-speed switch. Note that identical source code, DES encryption libraries, and MD5 authentication libraries were used for all experiments.

In all the microbenchmark experiments encryption was performed using a DES library in cipher block chaining (CBC) mode. The libarary used is libdes version 3.00 written by Eric Young. Authentication in all the microbenchmark experiments used the MD5 message digest algorithm. The library used is RSAREF version 2.0 from RSA Laboratories. The SSL tests were conducted using SSLRef 2.0 from Netscape Communications Corporation. This software uses RSAREF version 2.0 from RSA Laboratories to perform the public/private key operations for handshaking and key exchange, and, as already mentioned, uses libdes version 3.00 from Eric Young to perform encryption and RSAREF version 2.0 from RSA Laboratories to perform authentication.

The results reveal a number of interesting attributes of our Nexus secure communication infrastructure. Looking first at Figure 4, we note that for small messages, the underlying communication protocol (TCP vs. MPL) makes a bigger difference to performance than whether or not security is enabled. For a 10-byte message, unsecure MPL communication takes $108 \mu \mathrm{sec}$, while secure MPL takes $210 \mu \mathrm{sec}$ : 94 percent slower than unsecure MPL, but still a lot faster than both secure and unsercure TCP, which take 770 and 929 $\mu \mathrm{sec}$, respectively. These results emphasize the importance of using optimized low-level communication mechanisms when these are available.

For larger messages, encryption costs dominate communication time. Beyond 300 bytes, secure MPL is slower than unsecure TCP (but still considerably faster than secure TCP). Looking at Figure 5, we see that for messages larger 


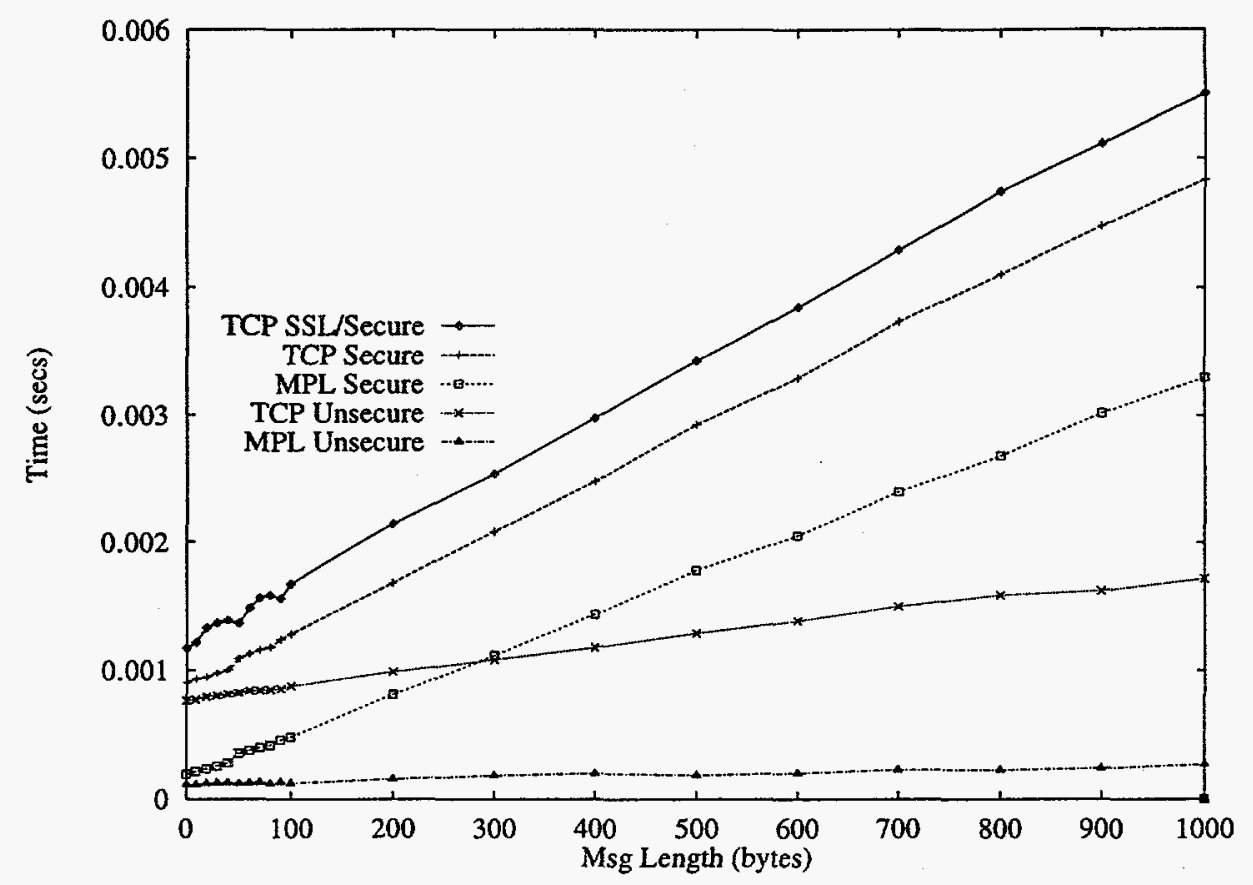

Figure 4. Microbenchmark results: See text for details

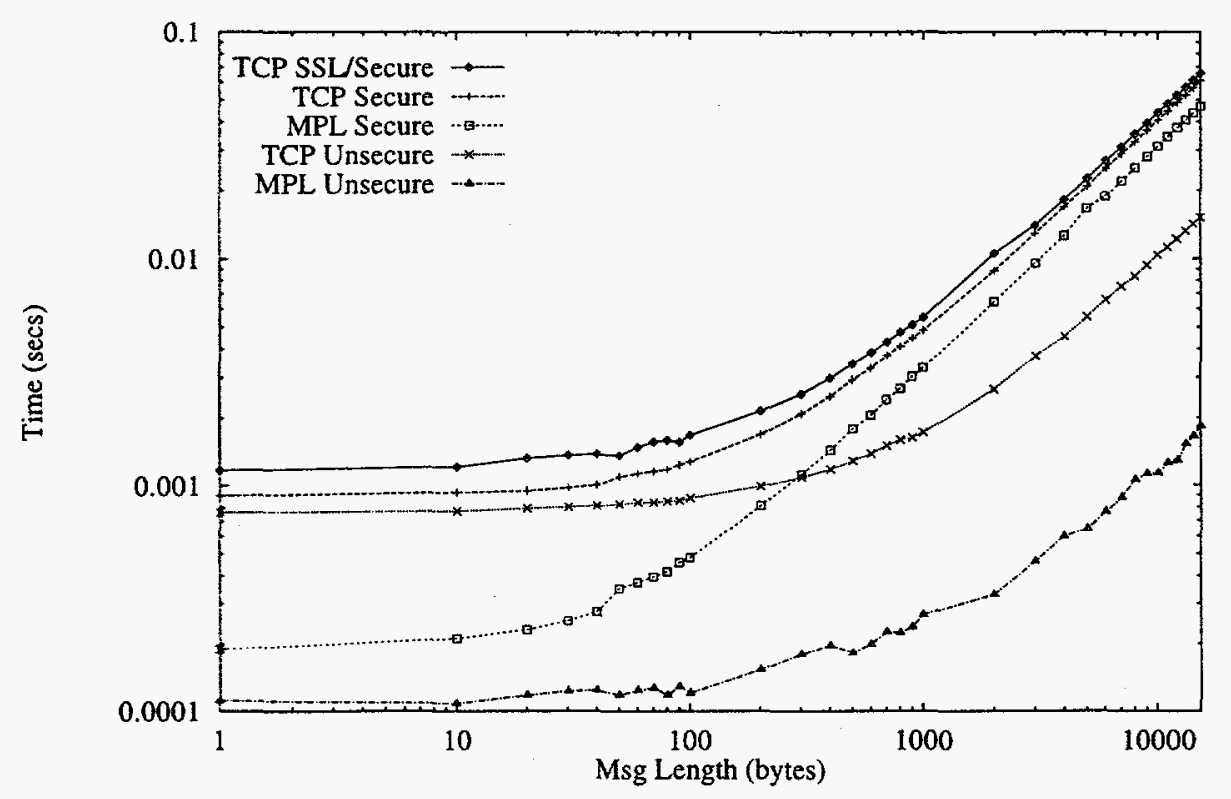

Figure 5. Microbenchmark results; note the use of log scales. See text for details 

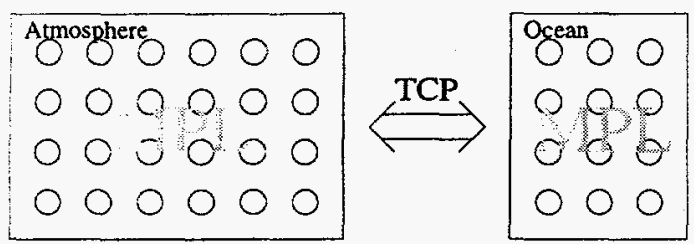

Figure 6. The Argonne/Wisconsin coupled ocean/atmosphere model in the configuration used for our multimethod communication experiments, showing the two IBM SP partitions.

than a few thousand bytes, secure MPL and TCP have essentially the same cost. This is because the communication costs for large messages are dominated by the limited performance of the DES encryption library; the Power 1 processor can encrypt and then decrypt data at only $0.33 \mathrm{Mbytes} / \mathrm{sec}$, far slower than the SP2 network.

Our results also reveal insights into the efficiency of SSLref. We see that for a 10-byte message, Nexus when using SSLref takes $1213 \mu \mathrm{sec}$ for a communication vs. 929 $\mu \mathrm{sec}$ when the same security mechanisms are integrated into Nexus. This $284 \mu \mathrm{sec}$ ( 31 percent) overhead is due to additional network L/O operations in the SSLref version, and extra copy operations.

\subsection{Application Results}

Our application study uses the FOAM fast oceanatmosphere model, designed to run at relatively low resolutions for multicentury simulations. This model uses MPI for communication and combines a large atmosphere model (the Parallel Community Climate Model [6]) with an ocean model (from U. Wisconsin). The two models execute concurrently and perform considerable internal communication. Every two atmosphere steps, the models exchange information such as sea surface temperature and various fluxes.

To provide a controlled environment for our experiments, we run the two model components not on two different computers but instead on distinct partitions of the Argonne SP2 (Figure 6). Communication between partitions is always performed by using TCP, this time utilizing the high-speed switch hence approximating a situation in which we have two computers connected by an ATM metropolitan area network. Communication within a partition may be performed by using either MPL or TCP; we present results for both cases. In all cases, user-level communication is achieved by using the MPI implementation that we have constructed by layering on top of Nexus [9]. (This layering adds an execu-
Table 1. Time per simulated day for the coupled ocean/atmosphere model, with different security modes and communication protocols on an IBM SP2

\begin{tabular}{|l|r|r|}
\hline Mode & $\begin{array}{r}\text { TCP time } \\
\text { (secs/day) }\end{array}$ & $\begin{array}{r}\text { MPL time } \\
\text { (secs/day) }\end{array}$ \\
\hline No secure & 854 & 574 \\
Coupler secure & 897 & 590 \\
All secure & 1459 & 1187 \\
\hline
\end{tabular}

tion time overhead of about 6 percent when compared with a "native" MPI.) No changes to the application program were required to run the different scenarios considered below.

Table 1 gives our results. We present results for three different scenarios: no encryption ("No secure"), encryption (ECB mode) only on communications between models ("Coupler secure"), and encryption (ECB mode) on all communications ("All secure"). In each case, we consider configurations in which either TCP or MPL are used within a partition.

Our results demonstrate the importance of a communications infrastructure that can both support the use of multiple low-level communication methods (MPL as well as TCP) and permit selective application of encryption. When using encrypted TCP for all communication, total time is 1459 seconds per simulated day. Allowing the use of MPL within a partition reduces execution time by 19 percent, to $1187 \mathrm{sec}-$ onds/day. Turning off encryption within partitions reduces execution time by a further 50 percent, to 590 seconds/day. The latter time is only 3 percent slower than when using no encryption at all.

\section{Related Work}

While there has been considerable earlier work on portable security mechanisms for distributed computing, issues relating to high-performance computing have received less attention.

The Secure Socket Library [12] (SSL) allows the programmer to associate different security mechanisms with different physical connections (sockets), but does not permit the use of specialized communication methods. In contrast, Nexus allows different security mechanisms to be associated with different logical connections, which furthermore can communicate with different low-level protocols.

Jaspan [14] describes the use of GSS-API to implement secure remote procedure calls. He reports an overhead of over 11 milliseconds for a secured RPC with no arguments; clearly, this work does not emphasize performance. 
Venugopal [28] describes a secure implementation of Parallel Virtual Machine, a popular message passing library. He uses a secure rsh for remote process creation and DiffieHellman key exchange to communicate a secret session key from the initial user process to all other processes. Encryption is enabled on a per-session basis, at the command line, although the programmer also has the option of specifying that a specific message should be secured using a particular technique. There is no support for associating a security mechanism with a particular logical connection.

The Prospero Resource Manager (PRM) [22] uses Kerberos mechanisms to provide secure process creation mechanisms for PVM. Depending on the level of security required, PRM can be configured to execute (a) only those programs whose executables reside in the PRM binaries directory (b) executables residing on the filesystem local to the site (c) local executables as well as those downloaded from remote sites from which jobs are submitted.

The $x$-kernel [24] and Horus [27] use protocol composition techniques to construct security enhanced versions of communication methods without the specialized "escape" used in Nexus. This approach introduces certain overheads but has high flexibility. We hope to explore its use in future work.

\section{Conclusions}

We have described the design and implementation of a secure communications infrastructure for high-performance distributed computing applications. This infrastructure integrates authentication, encryption, and data integrity mechanisms into the tools typically used to develop highperformance applications. These security-enhanced tools make it possible to run large-scale distributed applications in a secure manner, without any changes to the applications themselves. In addition, the tools provide hooks that programmers can use to manage explicitly the security mechanisms used for different communications. Experimental studies demonstrate that in a TCP/IP environment our performance is superior to that of SSLref, while in heterogeneous environments we can obtain significant performance advantages by employing multiple transport mechanisms and by enabling security mechanisms only when communicating selectively.

In future work, we propose to deploy these securityenhanced communication tools in a wide-area computing testbed that we are constructing, called GUSTO. This deployment will allow large-scale application experiments and hence provide feedback on how our security mechanisms work in practical situations. It seems certain that encryption performance will be a bottleneck in many situations. Hence, we will experiment with various performance enhancement techniques, including specialized protocols [1], parallel encryption algorithms $[20,21]$, and use of dedicated encryption processors. Another interesting direction for further work will be to investigate the feasibility of using the Metacomputing Directory Service to determine when secure communication mechanisms must be employed, for example because communication occurs over insecure network connections. Clearly one issue that will be important to address in this context is the authenticity of resource database entries.

\section{Acknowledgments}

We are grateful to Jonathan Geisler for his considerable assistance with the experiments reported in this paper, and to John Anderson, Robert Jacob, and Chad Schafer for making the coupled model available to us. This work was performed under the auspices of the Zipper secure communications library project, which is funded by the Mathematical, Information, and Computational Sciences Division subprogram of the Office of Computational and Technology Research, U.S. Department of Energy, under Contract W-31-109-Eng38.

\section{References}

[1] I. Agi and L. Gong. An empirical study of secure MPEG video transmissions. In Proc. Symp. on Network and Distributed System Security, pages 137-144. IEEE Computer Society Press, 1996.

[2] C. Catlett and L. Smarr. Metacomputing. Communications of the $A C M, 35(6): 44-52,1992$.

[3] T. DeFanti, I. Foster, M. Papka, R. Stevens, and T. Kuhfuss. Overview of the I-WAY: Wide area visual supercomputing. International Journal of Supercomputer Applications, 10(2):123-130, 1996.

[4] D. Diachin, L. Freitag, D. Heath, J. Herzog, W. Michels, and P. Plassmann. Remote engineering tools for the design of pollution control systems for commercial boilers. International Journal of Supercomputer Applications, 10(2):208218,1996

[5] T. L. Disz, M. E. Papka, M. Pellegrino, and R. Stevens. Sharing visualization experiences among remote virtual environments. In International Workshopon High Performance Computing for Computer Graphics and Visualization, pages 217-237. Springer-Verlag, 1995.

[6] J. Drake, I. Foster, J. Michalakes, B. Toonen, and P. Worley. Design and performance of a scalable parallel Community Climate Model. Parallel Computing, 21(10):1571-1591, 1995.

[7] S. Fitzgerald, I. Foster, C. Kesselman, G. von Laszewski, W. Smith, and S. Tuecke. A directory service for configuring high-performance distributed computations. Preprint, Mathematics and Computer Science Division, Argonne National Laboratory, Argonne, IIl., 1997.

[8] I. Foster, J. Geisler, C. Kesselman, and S. Tuecke. Multimethod communication for high-performance metacomput- 
ing applications. In Proceedings of Supercomputing '96. ACM Press, 1996.

[9] I. Foster, J. Geisler, and S. Tuecke. MPI on the I-WAY: A wide-area, multimethod implementation of the Message Passing Interface. In Proceedings of the 1996 MPI Developers Conference, pages 10-17. IEEE Computer Society Press, 1996.

[10] I. Foster, C. Kesselman, and S. Tuecke. The Nexus approach to integrating multithreading and communication. Journal of Parallel and Distributed Computing, 37:70-82, 1996.

[11] W. Gropp, E. Lusk, and A. Skjellum. Using MPI: Portable Parallel Programming with the Message Passing Interface. The MIT Press, 1994.

[12] K. Hickman. The SSL protocol. Internet Draft RFC, 1995.

[13] International Business Machines Corporation, Kingston, NY. IBM Load Leveler: User's Guide, September 1993.

[14] B. Jaspan. GSS-API security for ONC RPC. In Proc. Symp. Network and Distributed Systems Security, pages 144-151. IEEE Computer Society Press, 1993.

[15] C. Koelbel, D. Loveman, R. Schreiber, G. Steele Jr., and M. Zosel. The High Performance Fortran Handbook. The MIT Press, 1994.

[16] C. Lee, C. Kesselman, and S. Schwab. Near-realtime satellite image processing: Metacomputing in $\mathrm{CC}++$. Computer Graphics and Applications, 16(4):79-84, 1996.

[17] J. Linn. Generic security service application program interface. Internet RFC 1508, 1993.

[18] T. Maples and G. Spanos. Performance study of a selection encrytion scheme for the security of networked, real-time video. In Proc. 4th Intl. Conf. on Computer Communications and Networks, 1995.

[19] C. Mechoso et al. Distribution of a Coupled-ocean General Circulation Model across high-speed networks. In Proceedings of the 4th International Symposium on Computational Fluid Dynamics, 1991.

[20] E. Nahum. S. O'Malley, H. Orman, and R. Schroeppel. Towards high performance cryptographic software. In 3rdIEEE Workshop on the Architecture and Implementation of High Performance Communication Subsystems, 1995.

[21] E. Nahum, D. Yates, S. O'Malley, H. Orman, and R. Schroeppel. Parallelized network security protocols. In Proc. Symp. on Network and Distributed System Security, pages 145-154. IEEE Computer Society Press, 1996.

[22] B. C. Neumann and S. Rao. The Prospero resource manager: A scalable framework for processor allocation in distributed systems. Concurrency: Practice \& Experience, 6(4):339355,1994

[23] M. Norman, P. Beckman, G. Bryan, J. Dubinski, D. Gannon, L. Hernquist, K. Keahey, J. Ostriker, J. Shalf, J. Welling, and S. Yang. Galaxies collide on the I-WAY: An example of heterogeneous wide-area collaborative supercomputing. International Journal of Supercomputer Applications, 10(2):131-140, 1996.

[24] S. O'Malley and L. Peterson. A dynamic network architecture. ACM Transactions on Computing Systems, 10(2):110143,1992

[25] J. Steiner. B. Neumann, and J. Schiller. Kerberos: An authentication system for open network systems. In Usenix Conference Proceedings, pages 191-202. 1988.

[26] D. Stevenson, N. Hillary, G. Byrd, F. Gong, and D. Winklestein. Design of a key agile cryptographic system for OC$12 \mathrm{c}$ rate ATM. In Proc. Symp. Network and Distributed Sys- tems Security, pages 17-30. IEEE Computer Society Press, 1993.

[27] R. van Renesse, K. Birman, R. Friedman, M. Hayden, and D. Karr. A framework for protocol composition in Horus. In Proc. Principles of Distributed Computing Conf., 1995.

[28] N. Venugopal. The design, implementation, and evaluation of cryptographic distributed applications: Secure PVM. Technical report, University of Tennessee, Knoxville, Tenn., 1996. 